\title{
Muscles Cramp and Sleep Disturbances among Hemodialysis Patients
}

\author{
Gulshan Roy Chowdhury \\ Ph.D Scholar \& Faculty, College of Nursing ABVIMS \& Dr. R.M.L Hospital, New Delhi
}

\begin{abstract}
Muscle cramps are often experienced by the patients on hemodialysis that result in discomfort, shortened treatment times, and inadequate dialysis dose.

Up to $50 \%$ people with chronic renal failure undergoing dialysis suffer from muscle cramps, especially involving the lower limbs. The cramps can happen during dialysis or at home. The cramps in this type of patient are linked to depression, a decline in quality of life, and sleep disorders. The etiology of cramps in hemodialysis patients is not clear.

Muscle Cramping of the hands, feet, and legs is fairly common among patients onhemodialysis. Muscle Cramps are also associated with low bloodpressure. However, some Muscle cramping continues even after a normal bloodpressure. When patients are below dry weight, they also experience muscle cramps. Thesevere muscle cramps are experienced near the end of the dialysis and persisting for atime after dialysis is often due to dehydration. ${ }^{(1)}$

The most frequent sleep disorders seen in patients on dialysis are conditioned insomnia, excessive daytime sleepiness, obstructive or central sleep apnea, as well as restless legs syndrome (RLS) and periodic limb movement disorder. Many uremic and non-uremic factors are thought to participate in the pathogenesis of sleep disorders in these patients. The treatment of sleeping disorders includes nonpharmacological and pharmacological measures that can improve the functionality and quality of life in patients on dialysis. ${ }^{(2)}$
\end{abstract}

Key Words: Muscle Cramps, Nocturnal Muscle Cramps, Restless Leg Syndrome, Quality of Life, Intradialysis stretching exercise.

\section{Introduction}

Muscle cramps are a common complication of hemodialysis treatments, occurring in 33 to 86 percent of patients; resulting in the early termination of a hemodialysis session and are therefore an important cause of under-dialysis.

Muscle cramps may cause by changes in plasma osmolality and/or extracellularfluid volume. Increasing dialysis treatment time to reduce ultrafiltration rate, minimizing intra-dialytic weight gains, avoiding hypotension and using sodium modeling may reduce cramps during dialysis treatments.
Administration of hypertonic saline or dextrose and local heat and massage of the affected muscle may treat cramps occurring during dialysis treatment. (3)

The top 3 prioritized physical symptoms of insomnia, muscle cramps, and fatigue were analyzed in a recent study from the standpoint of their current state, barriers, and knowledge gaps impeding progress and a possible path forward. ${ }^{(4)}$

A case study by Appanraj.R, et.al (2015)on 20 patients concludes that by assuring the dry weight 
and monitoring the sodiumfrequently the episodes of muscle cramps can be controlled. ${ }^{(5)}$

Fiona Blytone, Vivienne Chuter and Joshua Burns (2012) conducted a study toknow about the night time muscle cramps. A survey was conducted to know the patient experience, treatment modalities and help seeking behavior.Leg cramps during the night time was experienced by almost all of the hemodialysispatients. Night time cramps are more severe than the day time cramps and can't be relievedby means of current therapies. $^{(6)}$

Allen RE, Kirby KA., 2012 conducted a study regarding the effect of nocturnal legcramps. Cramps arises mainly due to the associated disease conditions such as lumbarcanal stenosis, hemodialysis, vascular disease and other related medical conditions. Almostabout $60 \%$ of the adults are affected by the muscle cramps during the bed time. Thetreatment modalities mainly given for the muscle cramps are administering magnesium, vitamin $\mathrm{B}_{12}$, calcium channel blockers. Non-pharmacological therapies like stretching canalso be used for reducing muscle cramps. By the study it is clear that nocturnal leg crampsare common in adults. ${ }^{(7)}$

Nocturnal calf muscle cramps are associated with substantially reduced quality of sleep and reduced physical aspects of health-related quality of life. ${ }^{(8)}$

Keeping in mind the importance of this topic, a review was conducted to characterize the frequency, severity, and timing of dialysis-related cramps and the various types of sleep disturbances in hemodialysis patients. Dialysis-related cramps can be extremely painful and can compromise the efficacy of hemodialysis therapy.Moreover, sleep disturbances can have adverse affect on such patients. So the treatment modalities of muscle cramps and sleep disturbances are also reviewed to focus on the point that patients on maintenance hemodialysis can lead a comfortable life.

\section{Muscle cramps among hemodialysis patients}

Muscle cramps are painful, sudden and involuntary muscle contractions that typically occur in lower extremities.Cramps can occur during hemodialysis sessions, in between the sessions, and during sleep also.

Though pathogenesis and exact mechanism of hemodialysis associated muscle cramps are still unknown, one or more of the following factors including hypovolemia, hyponatremia, tissue hypoxia, hypomagnesemia, carnitine deficiency and elevated serum leptin levels may be involved in the development of intra-dialysis muscle cramping. ${ }^{(9)}$

The etiology of muscle cramps in maintenance hemodialysis patients is poorly understood and no clear evidence-based prevention or treatment strategies are there. Several factors including a low concentration of serum magnesium may play a role The prevalence of hypomagnesemia (concentration of $<0.7 \mathrm{mmol} / \mathrm{L}$ ) in maintenance hemodialysis patients ranges from $10 \%$ to $20 \%$. A low dietary intake of magnesium, use of medications that inhibit magnesium absorption (e.g. proton pump inhibitors), increased magnesium excretion (e.g., high-dose loop diuretics), and a low concentration of dialysate magnesiumare the causes of hypomagnesaemia. Out of various therapies been explored (e.g.quinine, carnitine, vitamin $\mathrm{E}$, gabapentin, biotin, exercise, and massage), most have limited effectiveness and some may be unsafe for kidney failure patients. ${ }^{(10)}$

The psychological impact of cramps is often overlooked. Patient's mood and quality of life is significantly affected by cramps. They are manifested by HD patients as a particularly severe individual symptom, also linkedwithdepression and poor quality of life. ${ }^{(11)}$ 
Sleep and health-related quality of life, depression and anxiety are some of the adverse effects of cramps. Intra-dialysis massage appears to be an effective way to address muscle cramping.

In a Cochrane review in 2012, Blyton et al. examined other non-pharmacological interventions to help cramp symptoms. These included: using night splints: avoiding heavy covers on the bed, and making changes to sleeping position. changes to footwear; taking horse chestnut seed extract; Nocturnal calf muscle cramps are associated with substantially reduced quality of sleep and reduced physical aspects of health-related quality of life. Intradialytic stretching exercises during the last two hours of haemodialysis help to reduce and prevent the muscle cramps. ${ }^{(12)}$

According to Morris, (2014) there was a significant difference in the mean level of muscle cramps before and after intervention. It was found that in the pre test $53.3 \%$ had severe muscle cramps, $46.7 \%$ had a moderate muscle cramps and after performing the intra dialytic stretching exercise it was found that $40 \%$ had no muscle cramps, $24.4 \%$ mild and $35.6 \%$ had moderate muscle cramps. ${ }^{(13)}$

A study supported by Lekha. J. Abraham.et.al (2017) was done on the effectiveness of Intra-dialytic stretching exercise on prevention and reduction of muscle cramps among patients undergoing hemodialysis in Coimbatore. She concluded that stretching exercise during the hemodialysis prevent the occurrence of muscle cramps and improves the quality of life among these patients. ${ }^{(14)}$

Patient in the intervention group reported cramping at home decreased by 1.3 episodes per week compared to 0.2 episodes per week in the control group $(p=.005)$. Patient reported cramping during dialysis decreased by 0.8 episodes in the intervention group compared to 0.4 episodes in the control group $(p=0.44)$. Intradialytic massage found to be an effective way to address muscle cramping. ${ }^{(15)}$

The importance of performance of stretching exercises for patient before dialysis to prevent cramps is recommended. There is clearly a need for effective education regarding recognition of individual patient need \& appropriate interventionstrategies in muscle cramps in dialysis patient \& nurses, in partnership with patient, relatives\& other health professional can help to empower the individual to manage their cramps. ${ }^{(16)}$

Hallegraeff et al. identified that nightly stretching before going to sleep reduces the frequency and severity of nocturnal leg cramps in older adults. ${ }^{(17)}$

Application of Sequential compression devices (SCD) to LE may prevent the generation of LE HDrelated cramping in a select group of patients. (SCD) improve venous return (VR). ${ }^{(18)}$

In a Survey on Dialysis-Related Muscle Cramping, a combination of decreasing fluid removal, raising the lower extremities, and massaging the extremities was found to alleviate dialysis cramps. ${ }^{(19)}$

\section{Sleep disturbances in hemodialysis patients}

In the general population, prevalence of insomnia ranges from $4 \%$ to $29 \%$. Whereas in the ESRD population, approximately $50 \%-75 \%$ of patients experience symptoms of insomnia. Chronic pain, stress, older age, dialysis shift, melatonin, and high PTH, allcontributes in the development of insomnia in ESRD patients, although the mechanisms are not yet fully known.

A higher prevalence of sleep disorders has been associated with female gender in HD patients in other studies. ${ }^{(20)}$

For overall physical and mental well-being sleep is important. Sleep disturbances can include irregularity in sleeping habits, difficulty falling asleep, 
early morning awakening, and frequent awakening at night, sleep apnea and restless leg syndrome. Sleep disturbances among dialysis patients are found to be related to duration of dialysis therapy, high levels of urea and/or creatinine, pain, disability and somatic complaints such as pruritus and bone pain. ${ }^{(21)}$

Insomnia is common in HD patients and is frequently associated with other disorders of sleep.

A cross-sectional study (2017) was conducted to determine the prevalence and risk factors of insomnia in chronic hemodialysis (HD) patients.Female sex and duration on dialysis are the two risk factors were found. Insomnia does not appear related to any biochemical or dialysis parameters. Increased attention should be given to the management of dialysis patients regarding the diagnosis and management of insomnia and associated sleep disorders. ${ }^{(22)}$

A study (2020)underlined that among renal (i.e. causesof ESRD and previous kidney transplantation) and dialyticparameters (i.e. type and time on dialysis), only dialysis shiftwas associated with sleep disorders. This association is dueto the link between the morning shift and insomnia. ${ }^{(23)}$

Daniel, et.al. (2020) concluded the prevalence of sleep disturbances and depression was higher among the maintenance hemodialysis patients receiving dialysis for more than 2 years and also with a frequency of twice a week. It may be inferred that by increasingthe frequency and the dosage of dialysis in patients the quality of sleep can be adequately improved and thereby it may lower the depressive state in patients. ${ }^{(24)}$

The patient often develops an imbalance in various metabolites and bioelements as a consequence of CKD or ESRD and dialysis. In addition, patients also develop anemia due to various reasons including blood loss directly from the dialysis, a decrease in the levels of erythropoietin and frequent blood tests. As iron is bound to hemoglobin, a decrease in the count of red blood cells leads to a decrease in hemoglobin levels thereby causing a decrease in the levels of iron, which have been implicated as the primary cause of RLS (Restless Leg Syndrome) and is directly linked to $\mathrm{CKD}$ and dialysis. ${ }^{(25)}$

Samara, et.al (2019) recommended implementing the appropriate interventions to address the problem of poor sleep quality among dialysis patients. Such intervention may include sleep hygiene education, physical exercise, and cognitive-behavioral therapy, among other pharmacological and nonpharmacological modalities. ${ }^{(26)}$

Insomnia in patients on maintenance dialysis can also be exacerbated by other commonly co-occurring sleep disorders such as sleep apnea and restless leg syndrome.

Pain is poorly managed in patients with kidney disease. Non-pharmacological and non-opioid analgesics should be first-line approaches for pain management. Opioid use should be minimized with careful monitoring and dose adjustment. In a recent review, evidence supports the use of physical activity and other non-pharmacologic therapies, either alone or with pharmacological therapies, for pain management.Non-opioid analgesics, including acetaminophen, topical analgesics, gabapentinoids, serotonin-norepinephrine reuptake inhibitors, and TCA may be considered based on pain cause and type, with careful dose considerations in kidney disease. NSAIDs may be used in CKD and ESKD for short durations with careful monitoring. (27)

Due to the burden of illness, those on maintenance dialysis often live lives of compromised quality, which includes managing multiple concurrent symptoms and the demands of dialysis. There are a number of studies which support the fact that nurse led CBT improves patient's general health problems 
like insomnia, fatigue, and QOL. Therefore, active involvement of experienced nurses in the counseling program should be considered. In addition, further research must be conducted into the implementation of the nurse-led cognitive-behavioral intervention to a broader spectrum of patients. Improving the QOL of hemodialysis patients is as important as increasing the length of their lives. ${ }^{(28)}$

\section{Conclusion}

Sleep disturbances are extremely common in hemodialysis patients. Subjective sleep complaints are sleep apnea syndrome, restless legs syndrome, and periodic limb movement disorder are much more prevalent than in the general population and reported in up to $80 \%$ of those surveyed. Therefore, the purpose of this article is to present an up-to-date review of the literature regarding sleep disturbances in hemodialysis patients with special emphasis on the numerous factors including muscle cramps potentially contributing to these problems. Non-pharmacological and pharmacological measures are included in the therapy of sleeping disorders and muscle cramps that can improve the functionality and quality of life in patients with ESRD (End Stage Renal disease).

Ethical Permission: It's a review article. So no need to sort the ethical clearence.

\section{Conflict of Interest: Nil}

\section{Source of Funding: Self}

\section{References}

1. Bordoni B, Sugumar K, Varacallo M. "Muscle Cramps", StatPearls Publishing; 2021 Janhttps://www.ncbi.nlm.nih.gov/books/ NBK499895/

2. Kosmadakis GC, Medcalf JF. "Sleep disorders in dialysis patients". Int J Artif Organs, 2008, Nov;31(11):919-27. doi: $10.1177 / 039139880803101101$. PMID:
19089793.

3. Canzanello, Vincent \&Burkart, John. "Hemodialysis-Associated Muscle Cramps". Seminars in Dialysis. 2007, 5. 299 - 304. 10.1111/j.1525-139X.1992.tb00234.x.

4. Flythe J.E., Hilliard T., Lumby E. Fostering innovation in symptom management among hemodialysis patients: paths forward for insomnia, muscle cramps, and fatigue. Clin J Am SocNephrol. 2019; 14:150-160.

5. Appanraj.R., Kumar, A., \& Usha; "Muscle cramps episodes among chronic renal failure patients who are on haemodialysis". International Journal of advances in case reports, 2015, 2 (13), 805-807.

6. Blyton F, Chuter V, Burns J. "Unknotting night-time muscle cramp: a survey of patient experience, help-seeking behaviour and perceived treatment effectiveness". J Foot Ankle Res. 2012 Mar 15;5:7. doi: 10.1186/1757-11465-7. PMID: 22420923; PMCID: PMC3361473.

7. Allen RE, Kirby KA. "Nocturnal leg cramps". Am Fam Physician. 2012 Aug 15;86(4):350-5. PMID: 22963024

8. Hawke, F., Chuter, V., \& Burns, J. (2013). "Impact of nocturnal calf cramping on quality of sleep and health-related quality of life". Quality of Life Research, 2021, 22(6), 1281-1286. from http://www.jstor.org/stable/24724316

9. BeladiMousavi SS, Zeraati A, Moradi S, Mousavi MB. "The effect of gabapentin on muscle cramps during hemodialysis: A double-blind clinical trial". Saudi J Kidney Dis Transpl 2015;26:11428.

10. Varghese, A., Lacson, E., Sontrop, J. M., et.al. “A Higher Concentration of Dialysate Magnesium to Reduce the Frequency of Muscle Cramps: A Narrative Review". Canadian Journal of Kidney Health and Disease. 2020 
11. Weisbord SD, Fried LF, Arnold RM et al. "Prevalence, severity, and importance of physical and emotional symptoms in chronic hemodialysis patients". J Am Soc Nephrol 2005; 16: 2487-2494

12. Blyton F, Chuter V, Walter KE, Burns J. "Non-drug therapies for lower limb muscle cramps". Cochrane Database Syst Rev. 2012 Jan 18; 1:CD008496. doi: 10.1002/14651858. CD008496.pub2.

13. Basemath. S.S. Morris, "Effect of Intradialytic Stretching Exercise on Muscle Cramps among Patients undergoing Haemodialsis". Tamil Nadu Nurses and Midwives Council Journal of Medical and Surgical Nursing, 2014, volume 2, 8-11.

14. Lekha.J., Abraham,E., \&Malarvizhi.G. "Effectiveness of Intradialytic Stretching exercise on muscle Cramps among patients undergoing haemodialysis". IOSR Journal of Nursing and Health Science, 2017, 6 (2), 47- 53.

15. Mastnardo, D., Lewis, J. M., Hall, K., Sullivan, C. M., Cain, K., Theurer, J., Huml, A., \& Sehgal, A. R. "Intradialytic Massage for Leg Cramps Among Hemodialysis Patients: a Pilot Randomized Controlled Trial". International journal of therapeutic massage \& bodywork, 2016, 9(2), 3-8. https://doi.org/10.3822/ijtmb. v9i2.305

16. Mohamed, M, Ahmed, A, Zead, SA. "Impact of stretching exercises protocol on reduction of muscle cramping during haemodialysis, among chronic renal failure patients". Al-AzharAssiut Med J. 2007;5(2). http://www.aamj.eg.net/ journals/pdf/1150.pdf. Accessed June 26, 2020.

17. Joannes M. Hallegraeff, Cees P. van der Schans, Renee de Ruiter, Mathieu H.G. de Greef, "Stretching before sleep reduces the frequency and severity of nocturnal leg cramps in older adults: a randomised trial", Journal of
Physiotherapy, 2012, Volume 58, Issue 1, Pages 17-22, ISSN 1836-9553, https://doi.org/10.1016/ S1836-9553(12)70068-1.

18. Ahsan M, Gupta M, Omar I, Frinak S, Gendjar S, Osman-Malik Y, Yee J. "Prevention of hemodialysis-related muscle cramps by intradialytic use of sequential compression devices: a report of four cases". Hemodial Int. 2004 Jul 1;8(3):283-6. doi: 10.1111/j.14927535.2004.01106.x. PMID: 19379428.

19. Punj, S., Enaam, A., Marquez, A., Atkinson, A. J., Jr, \&Batlle, D. "A Survey on DialysisRelated Muscle Cramping and a Hypothesis of Angiotensin II on Its Pathophysiology". Kidney international reports, 2020, 5(6), 924-926.

20. Hamzi MA, Hassani K, Asseraji M, El Kabbaj D. "Insomnia in hemodialysis patients: A multicenter study from morocco". Saudi J Kidney Dis Transpl; 2021 Apr 21]; 28:1112-8.

21. Williams SW, Tell GS, Zheng B, Shumaker S, Rocco MV, Sevick MA; "Correlates of sleep behavior among hemodialysis patients. The kidney outcomes prediction and evaluation (KOPE) study". Am J Nephrol. 2002 Jan-Feb; 22(1):18-28.

22. Hamzi MA, Hassani K, Asseraji M, El Kabbaj D. "Insomnia in hemodialysis patients: A multicenter study from morocco". Saudi J Kidney Dis Transpl. 2017 Sep-Oct;28(5):1112-1118. doi: 10.4103/1319-2442.215152. PMID: 28937071.

23. Santhosh Pai B H, Nithya Chandran, Dattatray Prabhu. "Sleeplessness in patients with endstagerenal disease undergoing dialysis therapy in a tertiary care center". International Journal of Contemporary Medical Research2020; 7(6):F5F7.

24. Daniel, V., Meetha, S., Jayakumar, M., \&Sekar, M. "Quality and adequacy of sleep among dialysis patients". Biomedicine, 2021, 40(4), 
543-546. https://doi.org/10.51248/.v40i4.338

25. Nigam, G., Camacho, M., Chang, E. T., \& Riaz, M. "Exploring sleep disorders in patients with chronic kidney disease". Nature and science of sleep, 2018, 10, 35-43. https://doi.org/10.2147/ NSS.S125839

26. Samara, A.M., Sweileh, M.W., Omari, A.M. et al. "An assessment of sleep quality and daytime sleepiness in hemodialysis patients: a crosssectional study from Palestine". Sleep Science Practice, 2019, 3, 4. https://doi.org/10.1186/ s41606-019-0036-4
27. Roy, Payel \& Weltman, Melanie \& Dember, Laura \& Liebschutz, Jane \& Jhamb, Manisha. "Pain management in patients with chronic kidney disease and end-stage kidney disease". Current opinion in nephrology and hypertension.2020, 29. 10.1097/MNH.0000000000000646.

28. Rohini T, Ezhilarasu P. "A mixed method research on sleep, fatigue, and quality of life among patients undergoing hemodialysis: A pilot study". Indian J Cont Nsg Edn [serial online] 2015 [cited 2021 Apr 21]; 16:50-6. 